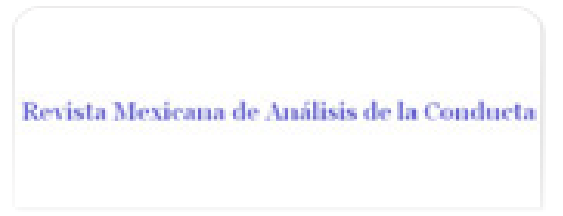

Revista Mexicana de Análisis de la Conducta ISSN: 0185-4534

editora@rmac-mx.org

Sociedad Mexicana de Análisis de la Conducta México

Vite, Ariel; López, Florente

Patrones de interacción madre-hijo en niños maltratados: un estudio observacional Revista Mexicana de Análisis de la Conducta, vol. 30, núm. 2, diciembre, 2004, pp. 163-179 Sociedad Mexicana de Análisis de la Conducta Guadalajara, México

Disponible en: http://www.redalyc.org/articulo.oa?id=59330203

- Cómo citar el artículo

- Número completo

- Más información del artículo

Página de la revista en redalyc.org

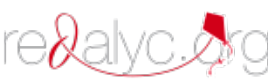

Sistema de Información Científica Red de Revistas Científicas de América Latina, el Caribe, España y Portugal Proyecto académico sin fines de lucro, desarrollado bajo la iniciativa de acceso abierto 


\title{
PATRONES DE INTERACCIÓN MADRE-HIJO EN NIÑOS MALTRATADOS: UN ESTUDIO OBSERVACIONAL
}

\author{
(MOTHER-CHILD INTERACTION PATTERNS IN MALTREATED \\ CHILDREN: AN OBSERVATIONAL STUDY) \\ ARIEL VITE Y FLORENTE LÓPEZ \\ UNIVERSIDAD NACIONAL AUTÓNOMA DE MÉXICO
}

\begin{abstract}
RESUMEN
Se compararon las interacciones de díadas madre-niño con historia de maltrato físico y díadas madre-niño sin historia de abuso infantil en dos situaciones: Académica y Juego Libre. Para ello se evaluaron el tiempo asignado a diversas conductas por la madre y el niño, la distribución de las consecuencias aplicadas por el adulto sobre las conductas negativas y positivas del niño; y el tipo de relaciones de contingencia. Los resultados indican que, aunque las díadas con historia de maltrato muestran mayores índices de conducta aversiva, de intercambios aversivos y de instrucciones que su contraparte control; los intercambios aversivos no muestran la extensión e intensidad característica del proceso de escalamiento. Estos hallazgos se discuten en el contexto de los modelos propuestos para la explicación de los patrones de interacción en el maltrato físico infantil.

Palabras clave: Maltrato infantil, indiscriminación materna, coerción, reforzamiento social.
\end{abstract}

1. Favor de enviar toda comunicación relacionada con el artículo a Ariel Vite correo electrónico: avite@servidor.unam.mx. 


\begin{abstract}
We compared mother-child interaction patterns of dyads of maltreated versus nonmaltreated children under two observational conditions: Academic and free-play. We evaluated the time assigned to different behavior categories by mother and child, the distribution of consequences by mothers over aggressive and positive behavior of child, and the mother-child contingency preferences. It was found that dyads with maltreatment background displayed higher indexes of aversive behavior, aversive exchanges and instructions than normal dyads. However, the scaling process as proposed by the coercion theory was not observed. These findings are discussed in the context of models oriented to the explanation of interactional patterns of children with physical maltreatment background.

Keywords: Children maltreatment, maternal indiscrimination, interaction patterns, coercion, social reinforcement.
\end{abstract}

En los últimos años, una línea de investigación sobre maltrato físico infantil estudia la naturaleza interactiva de los procesos involucrados en esta problemática y subraya la importancia del estudio de su desarrollo y mantenimiento (Ammerman, 1990; Reid, Taplin y Lorber, 1981; Wolfe, 1987). Los resultados de los estudios realizados bajo esta perspectiva coinciden en identificar una serie de comportamientos en la participación de la madre y del hijo en situaciones de interacción.

Con relación a la conducta materna, diversos estudios realizados tanto en ambientes naturales (Boshua y Twentyman, 1984; Lorber, Felton y Reid, 1984) como en condiciones de laboratorio (Oldershaw, Walter y Hall, 1986; Whipple y Webster-Stratton, 1991), encuentran que las madres maltratadoras muestran más conducta aversiva ( $\mathrm{Vg}$. regaños, desaprobaciones, gritos, amenazas) que las madres no maltratadoras. No obstante, otra serie de estudios señalan que la diferencia entre el comportamiento de estos tipos de madre no radica en su conducta negativa, sino más bien en el aspecto positivo. En otras palabras, las madres maltratadoras son menos positivas que las que no lo son. Sin embargo, investigadores como Lahey, Conger, Atkenson y Treiber (1984) y Boshua y Twentyman (1984) indican que no existen diferencias en los patrones de comportamientos maternales positivos ni en los negativos. Otros estudios reportan diferencias con relación a las instrucciones que las madres maltratadoras proporcionan a sus hijos, encontrándose que éstas aplican el doble de instrucciones que las madres normales (Cerezo, 1995; Cerezo, D’Ocon y Doltz, 1996; Cerezo y D’Ocon, 1995; Oldershaw, y cols., 1986).

Por lo que concierne al comportamiento infantil, se señala que los niños maltratados generalmente muestran comportamientos hostiles y de ais- 
lamiento en relación con sus pares (Cerezo, 1995). Pero también en este rubro se encuentran resultados discrepantes: mientras que un grupo de investigadores reportan altas tasas de conducta inadecuada ( $\mathrm{Vg}$. desobedecer, repelar) otros estudios señalan que en niños maltratados (Cerezo y D'Ocon, 1995; Oldershaw y cols., 1986) no existen diferencias significativas entre estos y niños sin problemas de maltrato (Burgess y Conger, 1978; Lahey, y cols., 1984; Lorber, y cols., 1984; Whipple y Webster-Stratton 1991). Lo mismo acontece con el comportamiento neutral o positivo: Si bien las tasas obtenidas muestran de manera consistente bajos valores para los niños maltratados, cuando se comparan con su contraparte, los niños normales, no se encuentran diferencias significativas (Cerezo y cols., 1996).

A pesar de tales discrepancias, los hallazgos de la investigación han permitido delinear propiedades de la regulación del comportamiento diádico en este tipo de parejas. En general, se consideran los siguientes factores: (a) Instrucciones: se dice que las madres maltratadoras carecen de habilidades para imponer sus órdenes, emplean una tasa elevada de instrucciones sin conseguir la obediencia del niño y, en consecuencia, provocan una serie de secuencias instruccionales del tipo orden-oposición-orden-oposición que propicia secuencias aversivas (Lorber y cols., 1984). (b) La conducta aversiva (Vg. regañar, amenazar, humillar) característica de las madres maltratadoras, está relacionada con tasas altas de conducta inadecuada por parte del niño (Vg. desobedecer, conducta oposicional, agredir) (Lorber y cols, 1984; Oldershaw y cols., 1986; Wipple y Webster-Stratton, 1991). (c) Conducta maternal inapropiada, la cual se manifiesta tanto en relación con la conducta prosocial del niño, como con la conducta negativa del mismo. De hecho, el comportamiento maternal inapropiado ha sido la variable más importante para clasificar a las madres con historia de maltrato físico (Cerezo y cols., 1996) y (d) Cuando se evalúa la relación entre conducta maternal inapropiada y conducta inapropiada del niño se observa que, conforme se repiten estos episodios, la probabilidad condicional de que la madre emita conducta inapropiada disminuye significativamente después de la conducta inapropiada del niño.

Además de la caracterización anterior, existen otras consideraciones que es necesario tomar en cuenta. En primer lugar, estos resultados han sido obtenidos considerando un solo miembro de la pareja, ya sea la madre o el niño, lo que proporciona una aproximación incompleta del fenómeno bajo estudio. Por otra parte, en los pocos trabajos que han empleado una perspectiva interaccional sus resultados señalan, de manera general, que las madres maltratadoras se comportan en forma aversiva hacia sus hijos de manera inoportuna. En otras palabras, sus intervenciones aversivas carecen de relación con lo que el niño hace o dice. Esta condición sitúa al niño en un contexto interactivo impredecible con importantes implicaciones para su conducta, tales como un elevado riesgo de psicopatología y, en particular, 
de conducta antisocial (Cerezo y cols., 1996; Reid, y cols., 1981). Ante esta situación se considera pertinente realizar estudios que incluyan a los dos miembros de la díada madre-niño, a fin de conocer con mayor detalle cómo se regulan sus intercambios y cuál es el manejo del reforzamiento positivo y negativo en dichos intercambios.

Por las razones previas en el presente estudio se evalúa a las díadas en una situación de interacción. El propósito específico fue comparar díadas madre-niño con historia de maltrato físico y díadas madre-niño sin historia de abuso infantil en dos situaciones, académica y juego libre. El objetivo fue establecer si existen diferencias en el tiempo asignado a diversas conductas por la madre y el niño, la distribución de las consecuencias aplicadas por el adulto sobre las conductas negativas y positivas del niño; y el tipo de relaciones de contingencia.

Se seleccionaron las dos condiciones de observación anotadas debido a que se ha reportado que representan situaciones extremas en las interacciones madre-niño. En la actividad de juego las madres son más didácticas y verbales, se observan ajustes en las interacciones entre ambos y parece propiciar un mayor involucramiento de la madre con sus hijos. Además, el juego del niño parece ser alentado por la estructuración paternal debido a su importancia educativa (Yogman, 1982, Pedersen, 1980; Power, 1985). Por otro lado, en la situación académica, los intercambios son menos frecuentes pero más conflictivos y se observa que los padres carecen de control sobre la conducta del niño en la realización de la actividad (Levin, Levy-Shift, Appelbaum-Peled, Katz y Komar, 1997; Rosemond, 1990).

\section{MÉTODO}

\section{Participantes}

Cinco díadas madre-hijo, con historia de maltrato físico, dos niñas y tres niños, y cinco díadas madre-niño sin historia de maltrato, dos niñas y tres niños, con un rango de edad entre los seis y los nueve años; con una media de 7.8 años para el grupo de maltrato y una media de 7.6 años para el grupo control. Las díadas con historia de maltrato físico infantil fueron canalizadas a través de la Unidad de Servicios de Apoyo a la Educación Regular (USAER) de la SEP y del DIF-Pachuca, por el equipo de profesionales encargados de detectar, confirmar y canalizar a los niños con este tipo de problemática. Las díadas sin historia de maltrato, determinadas como tal por el equipo de la USAER, se seleccionaron de una escuela primaria oficial del sur de la ciudad de México. En general, se intentó que ambos grupos fueran comparables en términos de edad, género, escolaridad y nivel socioeconómico. 


\section{Escenario}

Cada grupo de díadas fue video grabado en escenarios diferentes. Para las díadas con historia de maltrato físico infantil (Grupo Maltrato), las sesiones de observación se realizaron en una cámara de Gesell del Centro de Servicios Psicológicos de la Facultad de Psicología de la UNAM y en un cubículo ex profeso del DIF-Pachuca. Dichos escenarios se acondicionaron con una mesa de $80 \times 80 \mathrm{~cm}$. de una altura de $60 \mathrm{~cm}$., dos sillas, juguetes diversos (como muñecos, mecano, carros etc.), cuadernos para colorear y juegos de mesa, para cada una de las sesiones.

Para las díadas sin historia de maltrato (Grupo Control) las sesiones de observación se llevaron a cabo en el comedor familiar, contemplando los mismos materiales que las díadas con historia de maltrato físico.

\section{Materiales}

Videocámara, video casettes de $8 \mathrm{~mm}$, video reproductora VHS y televisión. Juguetes diversos, cuadernos para colorear, juegos de mesa, trípode, programa de registro, y Systat

\section{Procedimiento}

Las grabaciones de las díadas se llevaron a cabo por medio de una cámara de video sostenida por un trípode, colocado a tres metros de la mesa en la cual se encontraban la madre y el niño, para el Grupo de Maltrato, y en la esquina de la sala de la casa para el Grupo Control. Las diez díadas se observaron en 3 sesiones de 30 minutos, en dos condiciones de observación con una duración de 15 minutos cada una. De esta forma, se obtuvieron un total de 90 minutos de grabación, 45 por cada actividad, por cada díada. Las condiciones fueron las siguientes:

ACADÉMICA. Esta condición se programó con actividades supervisadas por la madre de acuerdo al nivel escolar de cada niño. De esta manera se pidió a las madres que supervisaran la tarea que correspondiera a su hijo en cada una de las sesiones a fin de que la elaboraran juntos.

JUEGO. En esta condición se dio la libertad al niño de elegir el tipo de juego, (Vg. damas chinas, palitos chinos, serpientes y escaleras, lotería, mecanos) dado que el objetivo de esta situación era observar las interacciones de cada díada, sin algún tipo de regla o demanda en particular.

Estas actividades se programaron de tal manera que tres sesiones de 15 minutos fueron para la actividad académica y tres sesiones con la misma duración para la de juego, efectuándose cada sesión de grabación en ese orden. 


\section{Sistema de Observación}

La codificación del total de los videos de las díadas se realizó por dos observadores independientes, el registro fue de tipo continuo con estimados en tiempo real. En una primera reproducción de los videos se registró la conducta de la madre y, en una segunda, la del niño. Con ese fin se utilizó un programa de cómputo en lenguaje Pascal diseñado para este tipo de estudios (Torres, Zarabozo y López, 1991) el cual posibilita la obtención de archivos individuales con la duración de las categorías y su secuencia a lo largo del tiempo de la sesión de observación. La codificación se realizó de la siguiente manera: se aplicó el mencionado programa el cuál generaba un archivo con las categorías y códigos de la misma. Para ello, se asignaba una tecla numérica del teclado de la computadora a cada categoría. De acuerdo al comportamiento observado se presionaba la tecla correspondiente, efectuándose el conteo de la duración de cada categoría, este procedimiento se continuó hasta finalizar con la sesión video grabada.

\section{Catálogo Conductual}

Se empleó un grupo de categorías utilizadas en el campo de investigación del maltrato físico infantil y se realizó una conjunción selectiva de las categorías propuestas en los trabajos de Amador, Pérez y Vite (1997), Mendieta y Vite (2000) y Parra y Vite (2002). Además, con el fin de adaptar las definiciones a las presentes condiciones de observación, se realizaron varias descripciones narrativas a partir de la observación de las video grabaciones de cinco díadas elegidas al azar. Posteriormente, se analizaron dichas descripciones y se conformó el catálogo conductual de la siguiente manera: El catálogo de conductas de la madre incluyó las categorías de Razonamiento (Ra), Aprobar (Ap), Instrucciones (In), Amenazar (Am), Desaprobar (De), Regañar (Re), Supervisar (Su), Reparar (Rp) y Otras (Ot). El catálogo de conductas niño incluyó Obedecer (Ob), Desobedecer (Ds), Repelar (Re), Realizar la actividad (Ra) y Otras (Ot). Véase el Apéndice 1 para la definición de las categorías.

\section{Concordancia entre observadores}

Se obtuvo un índice de concordancia entre observadores contrastando los dos registros de los catálogos conductuales obtenidos por los observadores independientes. Para ello se escogieron al azar un 30\% del total de sesiones tanto de la madre como del niño y de ambas condiciones, Académica y Juego. El índice de concordancia entre observadores se obtuvo con el Coeficiente Kappa de Cohen (Bakeman y Gottman, 1989) que corrige los acuerdos esperados por azar. 
Con dicho análisis se obtuvo una concordancia entre observadores de las conductas de las madres maltratadoras de .76 a .82 en la situación académica y de .80 a .84 en la de Juego. La concordancia entre observadores para las conductas de los niños con historia de maltrato fue de .80 a .86 en la situación académica y de .86 a .90 en la de Juego. Por lo que respecta a la concordancia de las madres del Grupo Control, esta fue de .82 a .91 en la situación académica y de .79 a .96 en la de Juego y la de los niños del Grupo Control fue de .85 a .92 en la situación académica y de .88 a .98 en la de Juego.

\section{Reducción de datos}

Para llevar a cabo el análisis de los datos se realizaron los siguientes pasos; en primer lugar los archivos en tiempo real fueron transformados en secuencias de comportamiento, de acuerdo con un muestreo temporal segundo a segundo. En segundo lugar, los dos archivos de datos obtenidos, uno de la madre y otro del niño, fueron combinados en uno solo, de forma que una secuencia de comportamiento fue la variable correspondiente a la madre y otra al hijo. Estos datos fueron analizados por medio del paquete estadístico Systat, con el propósito de obtener tanto los tiempos dedicados por las madres a los diferentes comportamientos considerados por el catálogo conductual, como para determinar las interacciones madre-niño significativas.

\section{Resultados}

A fin de determinar si las madres de los grupos Control y Maltrato difieren en la forma de participación en las situaciones Académica y de Juego se obtuvo el tiempo asignado por la madre a cada categoría conductual. Las categorías originales de Amenazar, Desaprobar y Regañar de la madre, fueron reagrupadas en una sola: NEGATIVAS. Los valores obtenidos, segundos de comportamiento, fueron transformados en minutos.

Con los valores obtenidos se aplicaron evaluaciones ANOVA para cada una de las categorías de la madre en un diseño mixto con Grupo como factor entre-sujetos y Condición como factor intra-sujeto. Se obtuvieron efectos significativos en las categorías Instrucciones, Negativas y Supervisión. En la primera se observaron efectos principales de Condición $(F[1,8]=14.27$, $p<.01)$. En la segunda se observaron efectos principales de Grupo ( $F[1,8]$ $=5.83, p<.05)$, Condición $(F[1,8]=6.20, p<.05)$ y de interacción Grupo por Condición $(F[1,8]=9.93, p<.05)$. En la tercera, Supervisión; se encontraron efectos principales de Grupo $(F[1,8]=5.82, p<.05)$. De lo anterior se puede concluir que las madres dedicaron más tiempo a emitir Instrucciones en la situación Académica que en la de Juego, pero que no hubo diferencias aso- 
ciadas a los grupos Control y Maltrato. Por otro lado, en lo que se refiere a la categoría de Conductas Negativas, las madres del Grupo Maltrato dedicaron mayor tiempo en la condición Académica que en la de Juego, mientras que las madres del grupo Control lo hicieron en la misma medida en ambas condiciones. También puede observarse que, en general, éstas últimas emplearon menor tiempo en la categoría Negativas que las madres del grupo Maltrato. Por último, la evaluación indica que las madres, en general, supervisaron más al niño en la condición Académica que en la de Juego. En la Tabla 1, se pueden consultar los detalles relacionados con estas comparaciones.

Tabla 1. Media del tiempo (en minutos) dedicado a cada una de las conductas por las madres de los grupos Maltrato y Control en las condiciones Académica y Juego. Entre paréntesis se presenta la desviación estándar.

\begin{tabular}{|l|c|c|c|c|}
\hline & \multicolumn{2}{|c|}{ Grupo maltrato } & \multicolumn{2}{c|}{ Grupo control } \\
\hline Categorías & Académica & Juego & Académica & Juego \\
\hline Razonamiento & 0.33 & 0.91 & 1.95 & 1.13 \\
\hline & $(0.58)$ & $(0.46)$ & $(0.58)$ & $(0.46)$ \\
\hline Aprobar & 0.59 & 0.40 & 0.50 & 0.39 \\
\hline & $(0.29)$ & $(0.27)$ & $(0.29)$ & $(0.27)$ \\
\hline Instrucciones & 4.11 & 2.55 & 3.11 & 1.7 \\
\hline & $(1.5)$ & $(1.19)$ & $(1.5)$ & $(1.19)$ \\
\hline Negativa & 1.86 & 0.41 & 0.27 & 0.44 \\
\hline & $(0.33)$ & $(0.25)$ & $(0.33)$ & $(0.25)$ \\
\hline Supervisar & 24.3 & 17.38 & 32.98 & 39.04 \\
\hline & $(4.20)$ & $(5.55)$ & $(4.20)$ & $(5.55)$ \\
\hline
\end{tabular}

Con el propósito de conocer cómo los niños distribuyen las conductas Activo y Aversiva (resultante de la combinación de Desobedecer con Repelar), a través del tiempo, se llevó a cabo un procedimiento similar al del análisis anterior: se sumaron los tiempos, en minutos, dedicados a cada una de ellas.

Como en el caso de las conductas de las madres, se aplicaron evaluaciones ANOVA para cada una de las categorías del niño en un diseño mixto con Grupo como factor entre-sujetos y Condición como factor intra-sujeto. Se encontraron efectos principales de Condición para las categorías Obedecer $(F[1,8]=7.17, p<.03)$ y Activo $(F[1,8]=14.96, p<.01)$. Las evaluaciones anteriores indican que los niños emplearon un mayor tiempo en la conducta Obedecer en la condición Académica que en la de Juego y que ocuparon mayor tiempo en Activo en la condición de Juego que en la Académica. Ninguna de estas diferencias se asoció con el factor Grupo. En la Tabla 2 se muestran detalles estadísticos de estas comparaciones. 
Tabla 2. Media del tiempo (en minutos) dedicado a las conductas Obedecer, Aversiva y Activo por niños de los grupos Maltrato y Control en las condiciones Académica y Juego. Entre paréntesis se presenta la desviación estándar.

\begin{tabular}{|l|l|c|c|}
\hline Conducta & Situación & Grupo maltrato & Grupo Control \\
\hline Activo & Académica & 29.1 & 34.79 \\
\hline & & $(2.77)$ & $(2.77)$ \\
\hline & Juego & 39.54 & 40.66 \\
\hline & & $(2.31)$ & $(2.31)$ \\
\hline Aversiva & Académica & 3.64 & 0.15 \\
\hline & & $(1.66)$ & $(1.66)$ \\
\hline & Juego & 0.56 & 0.01 \\
\hline & & $(0.37)$ & $(0.37)$ \\
\hline Obedecer & Académica & 1.46 & 0.52 \\
\hline & & $(0.36)$ & $(0.36)$ \\
\hline & Juego & 0.79 & 0.38 \\
\hline & & $(0.29)$ & $(0.29)$ \\
\hline
\end{tabular}

Para complementar lo anterior, se evaluó el comportamiento de la madre consecuente a la conducta positiva y negativa del niño. En la Tabla 3 se observa que las madres maltratadoras emplean más comportamientos negativos e instruccionales ante las conductas positivas y negativas infantiles que las normales. Además, proporcionan menos razonamientos y más consecuencias negativas ante comportamientos positivos, como el de activo, de sus hijos, respecto al empleado por las madres control.

Tabla 3. Media del tiempo (en minutos) del comportamiento maternal contingente a la conducta infantil de los grupos Maltrato y Control en las condiciones Académica y Juego.

\begin{tabular}{|l|cc|cc|c|c|c|c|c|c|}
\hline & & & \multicolumn{2}{|c|}{ Conducta } & \multicolumn{2}{|c|}{ Materna } & & \\
\hline Conducta & \multicolumn{2}{|c|}{ Positiva } & \multicolumn{2}{|c|}{ Negativa } & \multicolumn{2}{|c|}{ Instrucción } & \multicolumn{2}{|c|}{ Supervisar } & \multicolumn{2}{|c|}{ Razonamiento } \\
Infantil & Malt & Cont & Malt & Cont & Malt & Cont & Malt & Cont & Malt & Cont \\
\hline Obedecer & 4.6 & .8 & 8.2 & .8 & 22.6 & 4.8 & 51.6 & 45.8 & 0 & 2.2 \\
\hline Aversiva & 0 & 0 & 8.1 & .6 & 51.2 & 3.6 & 14.8 & 91.3 & 22 & .8 \\
\hline Activo & 43 & 44.8 & 271.6 & 9.2 & 249.4 & 229.8 & 3701 & 8107.8 & 52 & 133.8 \\
\hline
\end{tabular}


Por último, con el propósito de evaluar el tipo de relaciones de contingencia, es decir el efecto del reforzamiento y su papel en los intercambios de las díadas, se realizó un análisis de la relación entre el comportamiento de las madres y el de sus hijos. Para tal efecto se crearon matrices antecedenteconsecuente de la interacción madre-niño, con dicha matriz se efectuó un análisis Log-lineal. Un primer paso fue determinar si la estructura de relaciones es estable a través de los grupos y las condiciones. Los resultados obtenidos $\left(X^{2}{ }_{56}=4852.78 ; p>.0001\right)$ rechazan el modelo de homogeneidad, por lo que sugiere que el patrón de dependencias (conductas madre-niño) difiere entre los grupos, y entre las situaciones (Académica y Juego).

Posteriormente, se realizó el análisis de los residuos estandarizados para obtener los valores $Z$ críticos (+1.96). De manera exploratoria, con tal información se pueden determinar las transiciones significativas. Estas transiciones son las que ocurren en un nivel por encima del esperado por el azar, es decir, en las que se sugiere una relación de dependencia entre conducta antecedente y conducta consecuente.

En la Figura 1, se presentan el diagrama de estado de las interacciones de las díadas con historia de maltrato físico infantil y su grupo de control en la situación académica. En las primeras, se observan tres patrones en la actividad académica, en el primero se aprecia una probabilidad baja de obediencia infantil ante los razonamientos, instrucciones, aprobación y desaprobación materna. Además, se observa también una baja probabilidad de que ante dicho comportamiento del niño la madre proporcione razonamientos y aprobación, pero una probabilidad alta de que la madre desapruebe la desobediencia.

En el segundo patrón observado en el grupo Maltrato, se observa una baja probabilidad de que el niño desobedezca cuando la madre le da alguna instrucción, pero alta ante el regaño materno; cuando el niño desobedece existe una alta probabilidad de que la madre proporcione instrucciones y baja de regañarlo. Por lo que respecta al tercer patrón, se observa que cada vez que la madre desaprueba el niño repela y una probabilidad alta de presentar la misma conducta cuando la madre lo regaña. Cuando el niño repela se presenta una alta probabilidad de que la madre supervise, así como cuando este realiza la actividad (ver figura 1).

En comparación el grupo control, lado derecho de la figura, presenta dos patrones de interacción madre-niño. En el primero se observan probabilidades moderadas de que ante la obediencia infantil, la madre responda con razonamientos o aprobación y relativamente alta de que responda con instrucciones. En el segundo patrón se presentan probabilidades moderadas de que la madre proporcione razonamiento e instrucciones ante la desobediencia infantil.

Por lo que respecta a la situación de Juego, en la Figura 2 se presen- 
Grupo maltrato

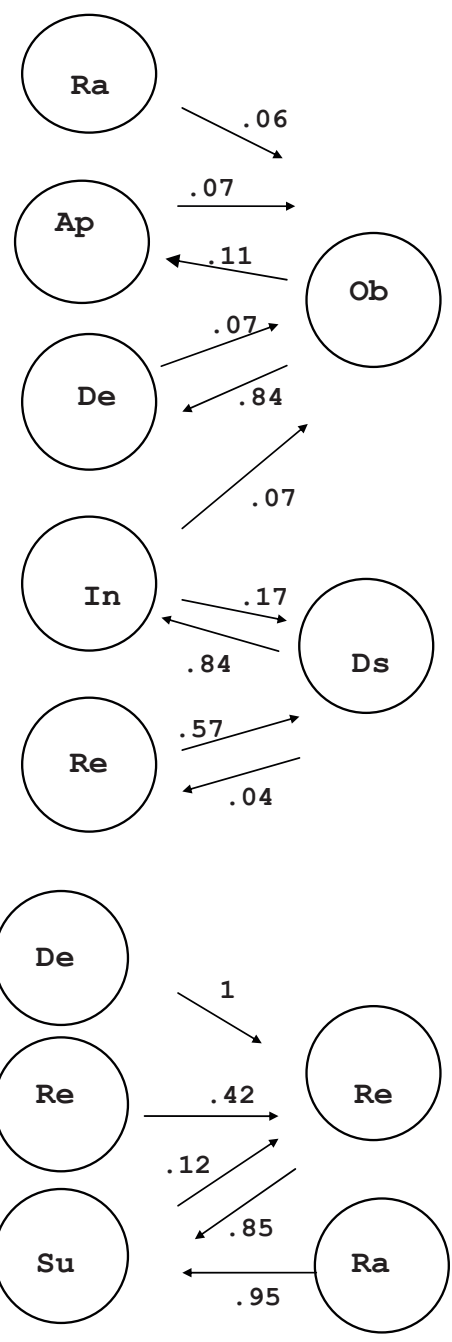

Grupo control
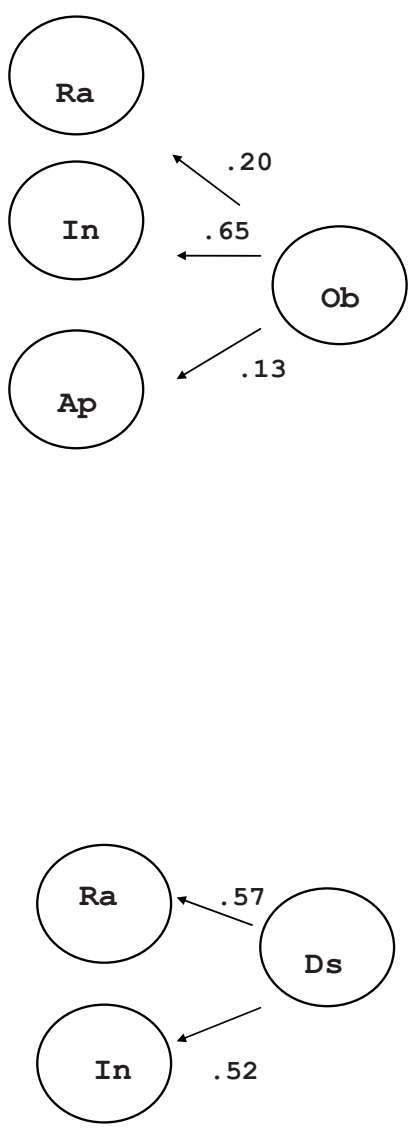

Figura 1.- Diagrama de estado de las conductas de las díadas de las dos muestras en la condición académica. Conductas de la madre: Ra = Razonamientos, $A p=$ Aprobar, In = Instrucciones, $D e=$ Desaprobar, $R e=$ Regañar, $\mathrm{Su}=$ Supervisar. Conductas del niño: $\mathrm{Ob}=$ Obedecer, $D s=$ Desobedecer, $R e$ $=$ Repelar, $R a=$ Realizar la actividad 
tan el diagrama de estado de los intercambios de las díadas con historia de maltrato y su grupo de comparación. En este diagrama se aprecia, para las primeras, una baja probabilidad de que el niño obedezca o desobedezca ante la instrucción materna, y una moderada probabilidad de que el niño desobedezca cuando la madre desaprueba la conducta desobediente.

Grupo maltrato

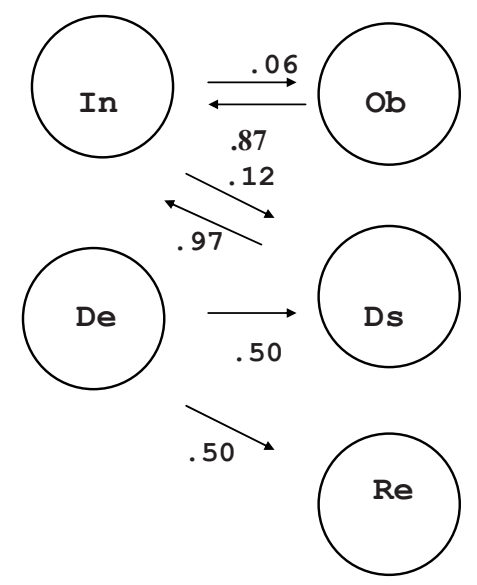

Grupo control
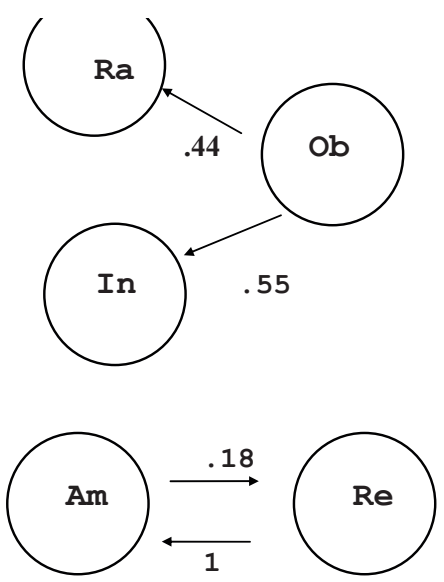

Figura 2.- Diagrama de estado de las conductas de las díadas de las dos muestras en la condición de juego. : Ra = Razonamientos, In = Instrucciones, $D e=$ Desaprobar, $R e=$ Regañar, $A m=$ Amenazar. Conductas del niño: $O b=$ Obedecer, $D s=$ Desobedecer, $R e=$ Repelar, $R a=$ Realizar la actividad

Por otra parte, ante la desobediencia infantil la madre observa una alta probabilidad de proporcionar instrucciones (lo que también ocurre ante la conducta de obedecer del niño) y, por último, cuando la madre desaprueba se presenta una probabilidad moderada de que el niño repele.

En lo que concierne a las díadas control, emergen dos patrones interactivos, en el primero se observan probabilidades moderadas de que ante la obediencia infantil la madre responda con razonamientos o instrucciones. En el segundo, se observa una probabilidad baja de que el niño repele ante una amenaza materna, pero cada vez que éste repela la madre lo amenaza.

\section{Discusión}

El interés principal de este estudio fue determinar si existen pautas espe- 
cíficas de comportamiento que caractericen las relaciones madre-hijo con antecedentes de maltrato del niño. Para ello, se compararon díadas sin antecedentes de abuso físico infantil con díadas con antecedentes de maltrato en condiciones que difieren en demandas normativas para el niño. Conviene resumir los hallazgos en las tres fuentes de datos evaluados: Tiempo asignado a las diferentes categorías conductuales por la madre y por el niño, distribución de tipo de consecuencias a las conductas del niño y dependencias entre las conductas de la madre y del niño.

En el primer análisis se observó que las madres maltratadoras exceden a las madres control en cuanto a los tiempos que invierten en conducta aversiva. Si bien el ordenamiento de las preferencias conductuales de la madre es similar en los dos grupos, la diferencia radica en los valores absolutos de tiempo dedicado a cada categoría conductual, particularmente en las de desaprobar, regañar e instrucciones. Respecto al análisis de las conductas de los niños se observó que los niños de ambos grupos ocupan casi el mismo tiempo en realizar la actividad tanto en la situación académica como en la de Juego. En cuanto a la conducta aversiva (desobedecer y repelar), se aprecian diferencias en la condición académica en donde los niños con historia de maltrato muestran un mayor tiempo en este tipo de comportamiento que su contraparte control. Sin embargo, en la situación de Juego no se observaron diferencias entre los dos grupos de niños en dicha conducta.

En cuanto al uso materno de consecuencias sociales, se observó que las madres maltratadoras de preferencia aplican consecuencias positivas a las conductas aversivas respecto a las conductas positivas del niño. Esta incongruencia sugiere un proceso de indiscriminación materna. Nuestros datos sugieren también que lo anterior es propio de las madres maltratadoras puesto que las madres normales siguen una tendencia opuesta a la anterior $y$, en particular, rara vez refuerzan conductas aversivas del niño.

Los resultados anteriores, en general, son similares a los obtenidos en diversos estudios (Cerezo y cols., 1996; Boshua y Twentyman, 1984; Cerezo y D'Ocon, 1995; Lorber y cols., 1984; Oldershaw y cols., 1986; Whaler y Dumas, 1986; Whipple y Webster-Stratton, 1995).

Por otra parte, un análisis central del presente estudio fue evaluar el intercambio conductual en las parejas. Para ello se estudiaron las dependencias entre la conducta de la madre y del niño y viceversa, es decir, relaciones en que la presencia de una conducta de un miembro predice la ocurrencia de una conducta particular del otro. Bajo estas condiciones se observó que las díadas madre-niño con historia de maltrato físico se involucran más que sus contrapartes control en intercambios aversivos ( $\mathrm{Vg}$. instrucciones-desobedecer, regañar-desobedecer, desobedecer-regañar, desaprobar-repelar, repelar-desaprobar, regañar-repelar). Esto se manifiesta en ambas situaciones, pero de manera más marcada en la situación de alta demanda (académica). 
Así mismo, en estas díadas se presentan probabilidades bajas de obediencia del niño ante las intervenciones de la madre así como una alta tasa de instrucciones maternales ante la obediencia infantil. Por otro lado, se observan pocas conductas positivas tales como razonamientos, aprobaciones ante la conducta de realizar la actividad y obedecer.

En comparación, el grupo de díadas sin historia de maltrato muestra un patrón de intercambios más positivo en ambas situaciones, académica y Juego, (Vg. obediencia-razonamientos, obediencia-aprobación, desobedienciarazonamientos, desobediencia-instrucciones). Sin embargo, al igual que las madres maltratadoras, emplean tasas altas de instrucciones. Además, provocan en el niño desaprobación cuando emplean amenaza y ante el repelar responden con amenazas. A pesar de estos comportamientos no se observan intercambios aversivos interdependientes.

De manera general, no obstante que las díadas madre-niño con historia de maltrato físico se involucran más que las madres control en episodios de coerción y castigan conductas positivas y refuerza negativas, no parecen que propicien uno de los procesos explicativos del maltrato, el escalamiento (Patterson, 1982; Reid, 1983); Reid y cols., 1981), es decir en el análisis de los datos generados, no se observaron intercambios aversivos, madre-niño mayores de 18 segundos, que de acuerdo a Reid (1983) es el criterio para propiciar un episodio de maltrato físico.

El análisis anterior lleva a plantear la existencia de un proceso, en el que tanto el reforzamiento positivo como el negativo mantienen la conducta aversiva infantil. Por un lado una madre puede ceder u obedecer a las demandas inherentes a la conducta oposicional del niño ante una instrucción. Por otro lado, es posible que la madre pueda estar preocupada por otros asuntos ajenos al niño, y algunas veces dispense atención de manera azarosa a la conducta del niño. Esta presumible condición de estímulos aversivos para el niño puede ser terminada a través de una respuesta coercitiva, porque esta acción es probable que sea seguido por un (predecible) contraataque materno.

Estos dos posibles procesos que toma el reforzamiento negativo, han sido conceptuados como hipótesis de "obediencia" (Patterson, 1982) e "indiscriminación materna" (Whaler y Dumas, 1986). Acorde con la primera, la conducta instruccional materna genera ambientes en los cuales se manifiesta conducta oposicional del niño y conducta aversiva de la madre. Esta situación se mantiene hasta que la madre desiste en su demanda; es decir, al ceder ante la conducta oposicional del niño la madre escapa de la situación aversiva. La segunda, propone que la conducta materna errática y desatenta genera un contexto interactivo, que para el niño es caracterizado como impredecible. Ante esto, la conducta desviada del niño logra tener la función de obtener una respuesta más predecible de la madre.

Los hallazgos obtenidos apoyan la pertinencia e importancia que para 
la investigación del maltrato tienen los estudios observacionales. Por un lado proporcionan evidencia respecto al proceso de interacción y, en consecuencia, permiten evaluar las hipótesis propuestas respecto al proceso de coerción que ocurre en el maltrato físico infantil. Por otro lado, los factores inmediatos relacionados con el maltrato infantil se encuentran en las situaciones de interacción cotidianas. Por consiguiente, el conocimiento de estos procesos de interacción, permitirá contribuir con elementos importantes para el diseño de estrategias de intervención para este tipo de población.

\section{REFERENCIAS}

Amador, A., Pérez, V., \& Vite, A. (1997). Programa de entrenamiento a padres para el manejo de problemas de conducta en niños: Una perspectiva de la interacción social. Enseñanza e Investigación en Psicología, 2, 139-159.

Ammerman, R. T. (1990). Etiological models of child maltreatment: A behavioral perspective. Behavior Modification, 14, 230-254.

Bakeman, J. \& Gottman, R. (1989). Observación de la interacción: Introducción al análisis secuencial. Madrid: Morata

Boshua, D. M., \& Twentyman, C. T. (1984). Mother-child interactional style in abuse, neglect and control groups: Naturalistic observations in home. Journal of Abnormal Psychology, 93, 106-114.

Burgess, R. L., \& Conger, R. D. (1978). Family interaction in abusive, neglectful and normal families. Child Development, 49, 116-1173.

Cerezo, M. A. (1995). El impacto psicológico del maltrato: Primera infancia y edad escolar. Infancia y Aprendizaje, 71, 135-157.

Cerezo, A., \& D'Ocon, A. (1995). Maternal inconsistent socialization: An interactional pattern in maltreatment children. Child Abuse Review, 4, 14-31.

Cerezo, A., D'Ocon, A., \& Doltz, I. (1996). Mother-child interactive patterns in abusive families versus nonabusive families: An observational study. Child Abuse and Neglect, 20, 573-587.

Lahey, B. B., Conger, R. D., Atkenson, B. M., \& Treiber, F. A. (1984). Parenting behavior and emotional status of physically abusive mothers. Journal of Consulting and Clinical Psychology, 52, 1061-1071.

Levin, J., Levy-Shift, R., Appelbaum-Peled, T., Katz, \& Komar, M. (1997). Antecedent and consequences of maternal involvement in child homework: A longitudinal analysis. Journal of Applied Developmental Psychology, 18, 207-227.

Lorber, R., Felton, D. K. \& Reid, J. B. (1984). A social learning approach to the reduction of coercive process in child abuse families: A molecular analysis. Advances of Behavior Research Therapy, 6, 29-45.

Mendieta, A. \& Vite, A. (2000). Obediencia infantil: El papel de los estilos disciplinarios maternales. Revista Mexicana de Psicología, 17, 1-17.

Oldershaw, L., Walters, C., \& Hall, K. (1986). Control strategies and noncompliance in abusive mother-child dyads: An observational study. Child Development, 57, 722-732. 
Parra, R. \& Vite, A. (2002). Modificación de estilos interactivos inmersos en el maltrato psicológico infantil. Integración: Educación y Desarrollo Psicológico, 7, 17-25

Patterson, G. R. (1982). A social learning approach: Vol. 3: Coercive family process. Eugene, OR: Castalia.

Pedersen, F. A. (Ed.). (1980). The father-infant relationship: Observational studies in the family-setting. New York: Praeger

Power, T. G. (1985). Mother-and father-infant play: A developmental analysis. Child Development, 56, 1514-1524.

Reid, J. (1983). Social interaccional pattern in families of abused and non abused children. En: Z. Maxler, M. Cummings, \& R. lannoti (Eds.), Altruism and aggression: Biological and social origins. (pp.238-255) New York: Cambridge University Press

Reid, J., Tapling, P. S., \& Loerber, R. (1981). A social interactional approach to the treatment of abusive families. En R. B. Stuart (Ed.), Violence behavior: Social learning approaches to prediction, management and treatment. (pp. 83-111). New York: Bruner/Mazel.

Rosemond, J. 1990). Ending the homework. Washington: Hassle McMeel.

Torres, A., Zarabozo, D., \& López, F. (1991). Registro observacional a través de computadora. Revista Mexicana de Análisis de la Conducta, 17, 147-161.

Whaler, R. G., \& Dumas, J. E. (1986). Maintenance factors in coercitive mother-child interactions: The compliance and predictability hypotheses. Journal of Applied Behavior Analysis, 19, 13-22.

Whipple, E. E., \& Webster-Stratton, C. (1995). The rol of parental stress in physical abusive families. Child Abuse \& Neglect, 15, 279-291.

Wolfe, D. (1987). Child abuse: Implications for child development and psychopathology. Beverly Hills, CA: Sage.

Yogman, M. W. (1982). Development of the father-infant relationship. En H. Fitzgerald, B, Lester, \& M. W. Yogman (Eds.). Theory and research in behavioral pediatrics Vol 1, (pp. 221-229), New York: Plenum Press.

\section{APÉNDICE}

Conductas de la madre

Razonamientos (RA): Verbalizaciones que señalan el por qué debe de realizar o no una actividad, indicando sus consecuencias.

Aprobar (AP): Expresiones verbales aprobatorias y/o movimientos verticales de cabeza que indican la aceptación de la conducta del menor.

Instrucciones (IN): Verbalizaciones que señalan la conducta que debe realizar el niño.

Amenazar (AM): Verbalizaciones para conducir a través de consecuencias aversivas la realización de una conducta.

Desaprobar (DE): Verbalizaciones y/o movimientos horizontales de cabeza para condenar la conducta del menor. 
Regañar (RE): Verbalizaciones en tono de voz alto, para expresar disgusto o enojo hacia la conducta del niño.

Supervisar (SU): Observar la actividad o conducta del niño, sin proporcionarle instigación física y/o verbal.

Reparar (RP): Verbalizaciones que indiquen el arrepentimiento de la madre por alguna acción aversiva dirigida al niño.

Otras (OT): Cualquier conducta no contemplada en las anteriores.

\section{Conductas niño}

Obedecer (OB): Llevar a cabo la instrucción dada por la madre.

Desobedecer (DS): No llevar a cabo las instrucciones proporcionadas por la madre.

Repelar (RE): Verbalizaciones que señalan contraposición a las instrucciones de la madre.

Realizar la actividad (RA): Involucrarse de manera directa en la tarea en cuestión.

Otras (OT): Cualquier conducta no contemplada en las anteriores. 